DR. ELISABETTA DEGASPERI (Orcid ID : 0000-0002-9899-4669)

DR. ENRICO GALMOZZI (Orcid ID : 0000-0001-8831-2401)

DR. MASSIMO IAVARONE (Orcid ID : 0000-0003-3493-6504)

DR. LUCA VALENTI (Orcid ID : 0000-0001-8909-0345)

DR. PIETRO LAMPERTICO (Orcid ID : 0000-0002-1026-7476)

Article type : Original

\title{
HEPATIC FAT - GENETIC RISK SCORE PREDICTS HEPATOCELLULAR CARCINOMA IN HCV CIRRHOTIC PATIENTS TREATED WITH DAAS
}

Elisabetta Degasperi1', Enrico Galmozzi ${ }^{1}$, Serena Pelusi ${ }^{2,3}$, Roberta D’Ambrosio ${ }^{1}$, Roberta Soffredini ${ }^{1}$, Marta Borghi ${ }^{1}$, Riccardo Perbellini ${ }^{1}$, Floriana Facchetti ${ }^{1}$, Massimo Iavarone ${ }^{1}$, Angelo Sangiovanni ${ }^{1}$, Luca Valenti ${ }^{2,3}$ and Pietro Lampertico ${ }^{1,3}$

${ }^{1}$ Foundation IRCCS Ca' Granda Ospedale Maggiore Policlinico - Division of Gastroenterology and Hepatology - CRC “A. M. and A. Migliavacca” Center for Liver Disease, Milan, Italy

${ }^{2}$ Department of Transfusion Medicine and Hematology, Fondazione IRCCS Ca' Granda Ospedale Maggiore Policlinico, Milan, Italy

${ }^{3}$ Department of Pathophysiology and Transplantation, University of Milan, Milan, Italy

Keywords: PNPLA3, MBOAT7, TM6SF2, liver tumor, steatosis

\section{Corresponding author}

Elisabetta Degasperi, MD

CRC “A. M. e A. Migliavacca” Center for Liver Disease,

Division of Gastroenterology and Hepatology

Fondazione IRCCS Ca' Granda Ospedale Maggiore Policlinico

This article has been accepted for publication and undergone full peer review but has not been through the copyediting, typesetting, pagination and proofreading process, which may lead to differences between this version and the Version of Record. Please cite this article as doi: $\underline{10.1002 / H E P .31500}$

This article is protected by copyright. All rights reserved 
University of Milan, Milan, Italy

Email: elisabetta.degasperi@unimi.it Phone: +390255035432 Fax: +390250320410

\section{Word count: 5613}

List of abbreviations: HCV: Hepatitis C virus, HCC: hepatocellular carcinoma; DAA: directacting antivirals, GRS: genetic risk score; PNPLA3: patatin like phospholipase domain containing 3; MBOAT7: membrane bound O-acyltransferase domain containing 7; TM6SF2: transmembrane 6 superfamily member 2; GCKR: Glucokinase Regulator; HR: Hazard ratio; SNP: single nucleotide polymporhism; NAFLD: non alcoholic fatty liver disease; HIV: human immunodeficiency virus; CPT: Child-Pugh-Turcotte; TE: transient elastography, LSM: liver stiffness measurement; SVR: sustained virological response; CR: complete response; US: ultrasound; AUROC: Area under the receiver operating characteristic curve; CI: confidence interval; Sn: Sensitivity; Sp: Specificity; LR: Likelihood ratio; PPV: positive predictive value; NPV: negative predictive value; BMI: body mass index; ALT: alanine aminotransferase; AFP: alpha-fetoprotein; INR: international standardized ratio; IQR: interquartile range; FIB-4: Fibrosis4 score; GGT: gamma-glutamyl-transferase

\section{Grant Support}

This study was funded in part by the following grants: "Ricerca Finalizzata RF2017/105-01"; "Ricerca Finalizzata RF2018/105-01"; "Ricerca Finalizzata RF2019/105-01"; Ricerca Finalizzata Ministero della Salute "RF-2016-02364358", Fondazione Sviluppo Cà Granda "PR-0316", the European Union Programme Horizon 2020 “No. 777377”.

\section{Conflict of Interest Statement}

Elisabetta Degasperi: Advisory Board: AbbVie; Speaking and teaching: Gilead, MSD, AbbVie Roberta D’Ambrosio: Advisory Board: AbbVie, Gilead; Speaking and teaching: AbbVie, Gilead, MSD, BMS; Research support: Gilead

Massimo Iavarone: Speaking and Teaching: Bayer, Gilead Science, Janssen, BTG, AbbVie; Consultant: BTG

Angelo Sangiovanni: Speaker Bureau: Bayer, Gilead Science, Janssen, BTG, AbbVie, Novartis; Advisory board: Tiziana Life sciences.

This article is protected by copyright. All rights reserved 
Luca Valenti: speaking: MSD, Gilead, AlfaSigma, AbbVie; consulting: Gilead, Pfizer, Astra Zeneca, Novo Nordisk, Diatech Pharmacogenetics, Intercept; research grants: Gilead.

Pietro Lampertico: Advisory Board/Speaker Bureau for: BMS, ROCHE, GILEAD SCIENCES, GSK, ABBVIE, MSD, ARROWHEAD, ALNYLAM, JANSSEN, SPRING BANK, MYR, EIGER

Other authors have nothing to disclose

\section{Writing assistance None}

\section{Authors' Contribution}

Concept and design: Elisabetta Degasperi, Pietro Lampertico, Luca Valenti

Data collection: Elisabetta Degasperi, Roberta D’Ambrosio, Roberta Soffredini, Marta Borghi, Riccardo Perbellini

Writing of article: Elisabetta Degasperi, Luca Valenti, Pietro Lampertico

Statistical analysis: Elisabetta Degasperi, Luca Valenti, Pietro Lampertico

Data management: Elisabetta Degasperi, Serena Pelusi, Massimo Iavarone, Angelo Sangiovanni

Genetic analysis: Enrico Galmozzi, Floriana Facchetti

Critical revision of the manuscript: Pietro Lampertico, Luca Valenti

\section{ABSTRACT}

Background \& Aims: Genetic factors and steatosis predispose to hepatocellular carcinoma (HCC) in chronic hepatitis C virus (HCV) patients, however their impact in cirrhotics cured by direct-acting antivirals (DAA) is still undefined. We assessed the association between a genetic risk score of hepatic fat accumulation (GRS), combining variants in PNPLA3, MBOAT7, TM6SF2, GCKR and HCC in DAA-treated patients. Approach \& Results: We considered 509 consecutive patients with $\mathrm{HCV}$ cirrhosis (defined histologically or when liver stiffness $\geq 12 \mathrm{kPa}$ ) treated with DAA. HCC was diagnosed according to international recommendations. GRS was calculated from the weighted impact of single variants on hepatic fat content quantified by $\mathrm{H}^{1}$-spectrometry in the general population (Dallas Heart Study). During a median follow-up of 43 (3-57) months after 
DAA start, 36/452 (8\%) patients developed de novo HCC, 4-year cumulative probability being 9\% (95\% CI 7-12\%). Male sex (HR 2.54, p=0.02), diabetes (HR 2.39, p=0.01), albumin (HR 0.35, $\mathrm{p}=0.001)$ and GRS score $>0.597$ (HR 2.30, p=0.04) were independent predictors of de novo HCC. In contrast, single genetic risk variants were not useful in stratifying HCC risk. The proportion of patients who developed HCC according to the combination of the independent risk factors ranged from $11 \%$ to $67 \%$. HCC recurred in $28 / 57$ (49\%) patients with previous history; diabetes and ethnicity were the only independent predictors of HCC recurrence. Conclusions: In a large cohort of HCV cirrhotic DAA-treated patients, GRS was associated with de novo HCC independently of classical risk factors including liver disease severity. These data suggest that hepatic fat lipotoxicity promotes HCC in this setting and may represent a target for chemoprevention. Combination of clinical and genetic predictors may improve HCC risk stratification.

\section{INTRODUCTION}

Direct-acting antivirals (DAA) have revolutionized treatment of chronic hepatitis $\mathrm{C}$ virus (HCV) infection, by providing excellent effectiveness with sustained virological response rates above 95\% in almost all patients [1]. In addition, the excellent safety profile has allowed extending treatment for patients with previous contraindications to Interferon (IFN)-based antiviral treatment. Despite HCV eradication results in reduced liver-related complications, patients who developed cirrhosis before HCV cure remain at risk of hepatocellular carcinoma (HCC) onset [2]. Consequently, current HCC international guidelines recommend long-life surveillance [3]. As the universal surveillance strategy in HCV-cured cirrhotic patients can result in elevated health cost burden, many efforts are currently aimed at identifying HCC predictors, in order to improve individual risk stratification and eventually develop personalized surveillance policies. Besides clinical predictors such as age, sex, metabolic co-factors (diabetes), more advanced liver stage, in recent years the expanding knowledge about the role of genetics in HCC predisposition prompted studies aimed at identifying the specific genetic determinants of HCC development. Many studies conducted in this field have showed robust association between single nucleotide polymorphisms (SNPs) and HCC risk especially in the setting of viremic HCV patients, but also in liver disease etiologies. In particular, common variants influencing hepatic fat accumulation and the risk of fatty liver disease have been associated with HCC development in several settings [4]. Concerning 
the specific variants identified, patatin like phospholipase domain containing 3 (PNPLA3) rs738409, membrane bound O-acyltransferase domain containing 7 (MBOAT7) rs641738, transmembrane 6 superfamily member 2 (TM6SF2) rs5842926 have provided the strongest evidence of increasing HCC risk [5-11]. The identification of these variants carries also a biological plausibility, as gene products are involved in regulation of fatty liver content, and metabolic co-factors have demonstrated strong association with risk of HCC development $[12,13]$. However, most studies have been conducted in the setting of HCV viremic or IFN-cured patients, so data about DAA-cured patients are still lacking. Moreover, an important limitation of currently available genetic studies relies in the low statistical power of a model based on a single-gene variant to predict HCC. We recently showed that a genetic risk score estimating the inherited predisposition to accumulate liver fat (GRS) resulting from combination of four major risk variants (in the PNPLA3, TM6SF2, MBOAT7, and Glucokinase Regulator [GCKR] genes) is associated with the severity of liver fibrosis in patients with non-alcoholic fatty liver disease (NAFLD) [14], suggesting that hepatic fat accumulation has a causal role in determining liver disease progression. However, little is known about the impact of hepatic fat in other liver disease etiologies or its potential association with liver-related events, such as development of HCC. With this in mind, we decided to evaluate the impact of the genetic predisposition to accumulate liver fat (estimated by the hepatic fat GRS) on HCC risk in a cohort of HCV cirrhotic patients cured by DAA.

\section{MATERIAL AND METHODS}

\section{Patient Population}

This single-center, retrospective study consecutively included HCV cirrhotic patients starting antiviral treatment with DAA between December 2014 and December 2016 and consenting to genetic testing. Patients were managed in the Gastroenterology and Hepatology Unit, Foundation IRCCS Ca' Granda Ospedale Maggiore Policlinico in Milan, Italy, which is a tertiary center for liver diseases. Main exclusion criteria were human immunodeficiency virus (HIV) co-infection, active HCC or atypical/uncharacterized liver nodules at baseline (DAA start) and Child-PughTurcotte (CPT) C score, whose treatment was not reimbursed by Italian National Health System outside the liver transplant waiting list, as previously described [12]. Cirrhosis was defined histologically (METAVIR F4) or non-invasively, by transient elastography (TE), with a liver stiffness measurement (LSM) cut-off $>11.9 \mathrm{kPa}$. Patients consented to make available their 
medical records for the study, which was approved by the local institutional review board and conformed to the 1975 Declaration of Helsinki ethical guidelines.

\section{Treatment efficacy and HCC surveillance}

Sustained virological response (SVR) was defined as undetectable HCV-RNA 12 weeks after the end of treatment, according to Abbott-Real Time PCR (lower limit of detection $12 \mathrm{IU} / \mathrm{mL}$ ).

HCC surveillance was performed by a 6-month abdominal ultrasound in patients with no HCC history; in patients with a previous HCC history, surveillance was performed by 6-month abdominal ultrasound (US) scan if they had achieved a complete response (CR) to a prior HCC treatment from $>2$ years, while a computed tomography every 3 months was performed in patients achieving a $\mathrm{CR} \leq 2$ years. $\mathrm{HCC}$ was diagnosed, staged and managed according to International recommendations [3].

\section{Genetic analysis}

DNA was purified from whole blood samples using the QIAmp blood Mini Kit (Qiagen, Mainz, Germany). Genotyping of the rs738409 $\mathrm{C}>\mathrm{G}$ (PNPLA3 I148M), rs58542926 C $>\mathrm{T}$ (TM6SF2 E167K), rs641738 C $>\mathrm{T}$ at the MBOAT7 locus, and rs1260326 C>T (GCKR P446L) was carried out with the commercial assays using the TaqMan single-nucleotide polymorphism genotyping allelic discrimination method (ABI prism StepOne Plus Real-Time PCR system, Applied Biosystems, Foster City, CA). Genotypes were determined by the SDS software (v.2.2, StepOne Plus; Applied Biosystems).

The hepatic fat GRS score was calculated by summing the number of at-risk alleles, weighted by their effect size on the hepatic fat content in a sample representative of the general population (Dallas Heart Study), as previously described [14].

\section{Statistical analysis}

Categorical variables were reported as frequencies (percentages) and continuous variables as median (range). Categorical variables were compared using the $\chi^{2}$ or the Fisher's exact tests; continuous variables were compared using the Student t-test, the Mann-Whitney $U$-test or the Kruskall-Wallis test, when appropriate. All tests were two-sided and used a significance level of 0.05. Logistic regression analysis was used to analyse the association between binary outcomes and multiple exposure variables. 
The optimal GRS cut-off values to predict HCC risk were identified using receiver operating characteristic (ROC) curves analysis. The area under the ROC curve (AUROC) as well as 95\% confidence interval (CI) were calculated under non-parametric (distribution free) assumption. The diagnostic performance of GRS to predict HCC was expressed as sensitivity ( $\mathrm{Sn}$ ), specificity (Sp), corresponding positive and negative likelihood ratios (LR+ and LR-), positive and negative predictive values (PPV and NPV), and AUROC.

The impact of risk factors on HCC development was evaluated by Kaplan-Meier estimates of the cumulative incidence rates; survival distributions were compared by univariate and multivariate Cox proportional-hazards models. All clinical variables included in the model were assessed at baseline (DAA start). Multivariate analysis included all significant $(\mathrm{p}<0.05)$ variables at univariate analysis. Patients were censored at last follow-up visit or HCC onset. Data handling and analysis were performed with StataView package (SAS Institute Inc., Cary, NC).

\section{RESULTS}

\section{Patient population}

Between December 2014 and December 2016, 565 HCV cirrhotic patients consecutively treated with DAA were enrolled; 56 did not consent to genetic testing, therefore, the final analysis included 509 patients. Patients characteristics are shown in Table 1. They were mostly (58\%) males, 471 (93\%) of Italian ethnicity, median age was 64 (28-87) years, body mass index (BMI) was $25(16-40) \mathrm{Kg} / \mathrm{m}^{2}, 99$ (20\%) patients had type 2 diabetes. HCV genotype was $1 \mathrm{~b}$ in 254 (50\%), median alanine aminotransferase ALT 71 (8-770), baseline LSM was 19.4 kPa (12.0-75.0); CPT score was A in 442 (87\%) of the patients, 57 (11\%) patients had a previous HCC history.

Unfavourable risk alleles for PNPLA3, MBOAT7, TM6SF2 and GCKR were carried by 275 (54\%), $363(71 \%), 46(9 \%)$ and 380 (75\%) of patients, respectively (Hardy Weinberg equilibrium $p>0.05$ ).

Patients' main baseline clinical and biochemical variables did not significantly differ across the genotypes of the four variants, except from higher alpha-fetoprotein (AFP) level and international standardized ratio (INR) in patients with PNPLA3 CC vs. CT/TT genotype and more HCV-1b patients in GCKR CC vs. CT/TT (Supplementary Table 1). Median GRS applied to patient population was 0.329 (Interquartile Range [IQR] 0.128-0.457).

\section{Antiviral treatment effectiveness}

This article is protected by copyright. All rights reserved 
Overall, 491 (96\%) patients achieved the SVR, $10(2 \%)$ had a viral relapse and $8(1.6 \%)$ were lost to follow-up before SVR timepoint (7 were retreated and achieved SVR). Treatment efficacy was not influenced by the genetic variants under study: indeed, GRS did not significantly differ according to the SVR status, being respectively 0.329 (IQR 0.128-0.457) in SVR vs. 0.361 (IQR $0.128-0.394)$ in non-SVR patients $(p=0.74)$.

\section{HCC development}

During 43 (3-57) months follow-up after antiviral treatment start, 64 (13\%) out of 509 patients developed HCC, with a 4-year cumulative incidence of 13\% (95\% CI 11-17\%); de novo HCC occurred in 36 patients without HCC history, while HCC recurred in 28 patients. The corresponding 4-year cumulative incidences of $\mathrm{HCC}$ were $9 \%$ (95\% CI 7-12\%) for de novo $\mathrm{HCC}$ and 51\% (95\% CI 27-63\%) for HCC recurrence (Figure 1). Complete description of HCC features is provided in the Supplementary Materials (Supplementary Text and Supplementary Table 2)

De novo HCC: clinical and genetic predictors

At univariate Cox regression analysis, carriage of the single genetic risk variants for hepatic fat accumulation was not significantly associated with HCC incidence, as well as GRS score when analyzed as a continuous variable (Supplementary Table 3). Since we hypothesized that the relationship between predisposition to hepatic fat accumulation - lipotoxicity and HCC was not linear, we examined whether we could identify a GRS score threshold that may be able to identify at risk patients with a high specificity. At AUROC curve analysis, the best GRS cut-offs for de novo HCC resulted GRS $>0.597$ [Sensitivity (Sn) 25\%, Specificity (Sp) 88\%, Positive Likelihood Ratio (LR+) 2.2, Negative Likelihood Ratio (LR-) 0.8, AUROC 0.52 (95\% CI 0.47-0.56)]. Of the whole cohort, 57 individuals $(12.6 \%)$ had a GRS $>0.597$. When considering in the univariate analysis GRS score (as a dichotomous variable) and clinical features, male gender, diabetes, baseline (DAA start) INR values, albumin, LSM, Fibrosis-4 (FIB-4) score and GRS $>0.597$ were associated with the incidence of de novo HCC (Table 2).

At multivariate Cox regression analysis that included all significant $(\mathrm{p}<0.05)$ variables at univariate analysis with the only exclusion of LSM values (due to limited data availability), male sex (Hazard Ratio [HR] 2.54, 95\% CI 1.1.5-5.63; $\mathrm{p}=0.02$ ), diabetes (HR 2.39, 95\% CI 1.20-4.74; $\mathrm{p}=0.01$ ), albumin concentration (HR 0.35, 95\% CI 0.19-0.64; $\mathrm{p}=0.001)$ and GRS $>0.597$ (HR 2.30,

This article is protected by copyright. All rights reserved 
95\% CI 1.03-5.11; $\mathrm{p}=0.04$ ) were independent predictors of de novo HCC (Table 2). Indeed, 4-year cumulative incidences of de novo HCC were 6\% (95\% CI 1-10\%) vs. 12\% (95\% CI 8-16\%) in males vs. females $(\mathrm{p}=0.01) ; 17 \%(95 \%$ CI 9-24\%) vs. 7\% (95\% CI 5-10\%) in diabetic vs. nondiabetic patients $(\mathrm{p}=0.001) ; 21 \%(95 \% 10-34 \%)$ vs. $7 \%(95 \%$ CI $4-11 \%)$ in patients with albumin $<$ or $\geq 3.5 \mathrm{~g} / \mathrm{dl}$ (lab normal albumin value was used as a cut-off) ( $<<0.001$ ), respectively. Concerning GRS score, cumulative incidence of HCC resulted 16\% (95\% CI 8-28\%) in patients with GRS $>0.597$ vs. $7 \%$ (95\% CI 5-11\%) in patients with GRS $\leq 0.597$ ( $p=0.01)$ (Figure 2A-D). By combining independent risk factors for HCC, 4-year cumulative incidence of HCC resulted $20 \%$ (95\% CI $12-28 \%$ ) in patients with two risk factors $(26.5 \%$ of the whole cohort) vs. $5 \%$ (95\% CI 3-7\%) in patients with less than two risk factors $(\mathrm{p}<0.0001)$. When evaluating presence of three contemporary risk factors $(1.3 \%$ of the whole cohort), the corresponding figures were $80 \%(95 \%$ CI $43-100 \%)$ vs. $8 \%(95 \% 6-11 \%)(\mathrm{p}<0.0001)$, while the small number of patients with four risk factors $(n=2)$ did not allow a further sub-analysis (Figure 3A-B). Finally, considering the proportion of patients with HCC development according to the multiple combinations of risk factors, HCC rates ranged between 11\% and 67\% (Figure 4).

\section{Recurrent HCC: clinical and genetic predictors}

At univariate Cox regression analysis, the presence of diabetes, BMI and Egyptian ethnicity were associated with HCC recurrence; on the other hand, the other single genetic variants and GRS score were not significant (Table 3). GRS was analyzed both as a continuous variable and by identifying a cut-off value at ROC curve analysis: GRS $<0.191$ [Sn 64\%, Sp 72\%, LR+ 2.3, LR0.5, AUROC 0.67 (95\% CI 0.53-0.78)].

At Cox regression multivariate analysis, diabetes (HR 2.78, 95\% CI 1.03-7.44, p=0.04) and ethnicity (Italian vs. Egyptian, HR 0.22, 95\% CI 0.05-0.91, p=0.03) were the only independent predictors of HCC (Table 3). The 4-year cumulative incidence of HCC was 88\% (95\% CI 64$100 \%)$ in diabetic patients vs. $45 \%(95 \%$ CI $25-55 \%)$ in non-diabetic patients $(\mathrm{p}=0.002)$ (Figure

5) and $100 \%$ vs. $48 \%$ (95\% CI 35-65\%) in Egyptian $v s$. Italian patients, respectively ( $\mathrm{p}=0.0003)$.

\section{DISCUSSION}

This single-center, retrospective-prospective study examined whether a GRS estimating the individual predisposition to accumulate fat in the liver predicted HCC onset in a cohort of 509 $\mathrm{HCV}$ cirrhotic patients, consecutively treated with antiviral therapy and followed-up for a median 
of 43 months. We confirmed that the GRS was associated with the incidence de novo HCC, independently of clinical predictors (diabetes, male sex, low pre-treatment albumin values). On the contrary, the genetic profile was not associated with HCC recurrence in patients with a previous history of HCC before antiviral treatment, the only independent predictors of HCC recurrence being the presence of diabetes and ethnicity.

To our knowledge, this is the first study investigating the role of genetic predisposition towards hepatic fat accumulation to predict $\mathrm{HCC}$ after $\mathrm{HCV}$ eradication, either as single risk variants or combined in a genetic risk score, in order to improve tumor risk stratification in HCV cirrhotic patients cured by DAA. As patients with de novo or recurrent $\mathrm{HCC}$ represent two distinct patient populations with regard to tumor history and risk, they have been analyzed separately.

Concerning the incidence of de novo HCC, the identified classical independent predictors, that is diabetes, male sex and albumin values, are in line with main literature results. Male sex has promptly been recognized as a risk factor for the development and progression of HCV-related liver disease to clinical complications [15]. Reduced circulating albumin concentration has been associated with de novo $\mathrm{HCC}$ in a recent multicentre Italian study enrolling 2,249 HCV cirrhotic patients treated with DAA. Low albumin values, together with reduced platelet count, which was identified by the same study as another independent predictor of HCC, represent a surrogate marker of more advanced liver diseases, meaning that de novo HCC risk is increased in patients treated for HCV infection at a more advanced disease stage [16]. The presence of advanced liver disease at baseline, as indicated by low platelet count, increased liver stiffness and/or portal hypertension, was found by all studies conducted in the DAA era as a key factor affecting HCC risk after viral cure [17-19]. Finally, diabetes had already been identified as HCC predictor in previous studies conducted in the IFN treatment era $[2,13]$; these data were confirmed also the patient populations treated with DAA [12]. Considering more broadly the role of metabolic cofactors, a French study conducted in $1,270 \mathrm{HCV}$ cirrhotic patients treated with DAA found that gamma-glutamyl-transferase (GGT) values were independently associated with HCC. Indeed, GGT is a marker of metabolic syndrome, although a potential role of alcohol intake and exposure to environmental toxins cannot be excluded [18]. BMI as marker of metabolic co-factor has been also included in a recent model to estimate HCC risk after SVR, developed in Veteran American patient population [20].

In this study, we focused on the impact of the genetic predisposition to hepatic fat accumulation, as a possible mediator of the effect of metabolic risk factors in determining the increased risk of 
$\mathrm{HCC}$ in a cohort of patients with cirrhosis, who mostly cleared the viral infection after DAA therapy. The four variants considered were identified in genome wide association studies as key determinants of hepatic steatosis and the risk of fatty liver disease. Although through different mechanism, their carriage results in increased predisposition to accumulation of fat within intracellular lipid droplets in hepatocytes [21-38]. The weighted hepatic fat GRS in which we combined these four variants was developed in a sample representative of the general population, where hepatic fat content was measured by the gold standard, that $\mathrm{H}^{1}$-spectrometry by nuclear magnetic resonance, and validated in multiple cohorts of at-risk individuals [14]. Importantly, it was demonstrated GRS correlated with liver fibrosis development in individuals with metabolic risk factors, suggesting hepatic fat accumulation has a causal role in liver disease pathogenesis and may represent a therapeutic target [4,14]. Variants in PNPLA3, TM6SF2 and MBOAT7 have been also identified as HCC predictors both in patients with steatohepatitis and HCV-related liver disease [5-11], but their role has never been evaluated in cirrhotic patients after HCV eradication. The main result of the present study was that, while the impact of single variants was not significant, the GRS was associated with the incidence of de novo HCC after DAA treatments, independently of classical risk factors and of the severity of liver disease. By combining the genetic risk score together with clinical predictors of HCC, in patients carrying at least two risk factors the cumulative incidence of de novo HCC was four-fold higher compared to patients carrying $<2$ risk factors $(20 \%$ vs. $5 \%$ at 4 years). This figure corresponds to a de novo HCC incidence of $5 \%$ per year in high-risk patients, that almost doubles the average 2-3\% reported in literature for cirrhotic patients after SVR [11,15,17,38-39]. As a consequence, combination of genetic and clinical risk factors could lead to identify a subset of cirrhotic patients (about one in four of the present cohort) carrying a high-risk of de novo $\mathrm{HCC}$, who could benefit from a personalized HCC surveillance program.

On the contrary, when analysing patients with a previous HCC history, we were not able to find any influence of genetic predisposition to accumulate liver fat on the risk of HCC recurrence, although we defined a specific cut-off of GRS for this patient subset. This could be due to the limited sample size of patients with previous HCC history $(n=57)$; in addition, tumor risk in these patients, who developed HCC during chronic viral replication, significantly differs from patients without any previous HCC and, consequently, predisposition of hepatic fat accumulation and altered hepatocellular lipid handling could not have a clinically meaningful impact on disease recurrence after curative treatments. 
Strengths of our work are the large patient population, consecutively enrolled with a consistent and systematic protocol of evaluation of demographic and clinical data and the long follow-up (median 43 months). Limitations include the lack of systematic assessment of the prevalence of fatty liver in the study cohort to confirm that the impact of GRS on HCC risk was mediated by altered hepatic lipid metabolism. However, the relationship between the GRS and hepatic fat was previously evaluated in multiple cohorts [14] as the amount of liver fat, but not the detrimental impact of altered lipid metabolism and lipotoxicity, tend to decrease with liver fibrosis severity. Furthermore, patients were mostly affected by compensated liver disease stage (CPT score A); this fact could have prevented us from observing an adequate number of HCC events, as decompensated patients (CPT B-C) carry an increased HCC risk compared to CPT A patients. However, according to the drug reimbursement policy in Italy, patients with decompensated cirrhosis CPT score C could not be treated if not waitlisted for liver transplant. Small numbers of patients with a previous history of HCC could also prevent us from the possibility to observe any association with genetic factors. As the cut-off of the hepatic fat content GRS for detection of HCC was derived from the ROC curve analysis in our patient population, it still requires external validation. In addition, results may not apply to populations characterized by a different risk profile or to other ethnicities; as we directly genotyped the four genetic variants of interest in the study cohort, unfortunately we were not able to perform more extensive genotyping allowing for a genetic estimation of ethnicity.

In conclusion, in a large cohort of HCV cirrhotic patients treated with DAA, we showed that a high value of a validated GRS estimating the inherited predisposition to accumulate fat in the liver was an independent risk factor for incidence of de novo HCC after DAA treatment. These data suggest that hepatic fat accumulation and lipotoxicity favour HCC development in this clinical setting. Therefore, the combination of clinical and genetic predictors could allow for a better HCC risk stratification in patients with $\mathrm{HCV}$-related cirrhosis treated with DAA, in order to develop individualized HCC surveillance strategies. On the other hand, drugs and lifestyle approaches targeting hepatic fat accumulation may reduce HCC incidence in individuals at higher risk.

\section{REFERENCES}

1) European Association for the Study of the Liver. EASL Clinical Practice Guidelines: Recommendations on Treatment of Hepatitis C 2018. J Hepatol 2018;69:182-236

This article is protected by copyright. All rights reserved 
2) van der Meer AJ, Feld JJ, Hofer H, Almasio PL, Calvaruso V, Fernández-Rodríguez CM, et al. Risk of cirrhosis-related complications in patients with advanced fibrosis following hepatitis C virus eradication. J Hepatol 2017;66:485-493

3) European Association for the Study of the Liver. EASL Clinical Practice Guidelines: Management of hepatocellular carcinoma. J Hepatol 2018;69:182-236

4) Romeo S, Sanyal A, Valenti L. Leveraging Human Genetics to Identify Potential New Treatments for Fatty Liver Disease. Cell Metab. 2020;31:35-45

5) Yang J, Trépo E, Nahon P, Cao Q, Moreno C, Letouzé E, et al. PNPLA3 and TM6SF2 variants as risk factors of hepatocellular carcinoma across various etiologies and severity of underlying liver diseases. Int J Cancer. 2019;144:533-544

6) Falleti E, Cussigh A, Cmet S, Fabris C, Toniutto P. PNPLA3 rs738409 and TM6SF2 rs58542926 variants increase the risk of hepatocellular carcinoma in alcoholic cirrhosis. Dig Liver Dis. 2016;48:69-75

7) Trépo E, Nahon P, Bontempi G, Valenti L, Falleti E, Nischalke HD, et al. Association between the PNPLA3 (rs738409 C>G) variant and hepatocellular carcinoma: Evidence from a meta-analysis of individual participant data. Hepatology 2014;59:2170-7

8) Sato M, Kato N, Tateishi R, Muroyama R, Kowatari N, Li W, et al. Impact of PNPLA3 polymorphisms on the development of hepatocellular carcinoma in patients with chronic hepatitis C virus infection. Hepatol Res. 2014;44:E137-44

9) Nakaoka K, Hashimoto S, Kawabe N, Nitta Y, Murao M, Nakano T, et al. PNPLA3 I148M associations with liver carcinogenesis in Japanese chronic hepatitis C patients. Springerplus. 2015;4:83

10) Valenti L, Motta BM, Soardo G, Iavarone M, Donati B, Sangiovanni A, et al. PNPLA3 I148M polymorphism, clinical presentation, and survival in patients with hepatocellular carcinoma. PLoS One. 2013;8:e75982

11) Donati B, Dongiovanni P, Romeo S, Meroni M, McCain M, Miele L, et al. MBOAT7 rs641738 variant and hepatocellular carcinoma in non-cirrhotic individuals. Sci Rep. $2017 ; 7: 4492$

This article is protected by copyright. All rights reserved 
12) Degasperi E, D'Ambrosio R, Iavarone M, Sangiovanni A, Aghemo A, Soffredini R, et al. Factors Associated With Increased Risk of De Novo or Recurrent Hepatocellular Carcinoma in Patients With Cirrhosis Treated With Direct-Acting Antivirals for HCV Infection. Clin Gastroenterol Hepatol. 2019;17:1183-1191

13) El-Serag HB, Kanwal F, Richardson P, Kramer J. Risk of hepatocellular carcinoma after sustained virological response in Veterans with hepatitis $\mathrm{C}$ virus infection. Hepatology 2016;64:130-7

14) Dongiovanni P, Stender S, Pietrelli A, Mancina RM, Cespiati A, Petta S, et al. Causal relationship of hepatic fat with liver damage and insulin resistance in nonalcoholic fatty liver. J Intern Med. 2018;283:356-370

15) Poynard T, Bedossa $P$, Opolon $P$. Natural history of liver fibrosis progression in patients with chronic hepatitis C. The OBSVIRC, METAVIR, CLINIVIR, and DOSVIRC groups. Lancet 1997;349:825-32

16) Calvaruso V, Cabibbo G, Cacciola I, Petta S, Madonia S, Bellia A, et al. Incidence of Hepatocellular Carcinoma in Patients With HCV-Associated Cirrhosis Treated With Direct-Acting Antiviral Agents. Gastroenterology. 2018;155:411-421

17) Conti F, Buonfiglioli F, Scuteri A, Crespi C, Bolondi L, Caraceni P, et al. Early occurrence and recurrence of hepatocellular carcinoma in HCV-related cirrhosis treated with directacting antivirals. J Hepatol 2016;65:727-33

18) Nahon P, Layese R, Bourcier V, Cagnot C, Marcellin P, Guyader D, et al. Incidence of Hepatocellular Carcinoma After Direct Antiviral Therapy for HCV in Patients With Cirrhosis Included in Surveillance Programs. Gastroenterology. 2018;155:1436-1450

19) Piñero F, Mendizabal M, Ridruejo E, Herz Wolff F, Ameigeiras B, Anders M, et al. Treatment with direct-acting antivirals for HCV decreases but does not eliminate the risk of hepatocellular carcinoma. Liver Int 2019;39:1033-1043

20) Ioannou GN, Green PK, Beste LA, Mun EJ, Kerr KF, Berry K. Development of models estimating the risk of hepatocellular carcinoma after antiviral treatment for hepatitis C. J Hepatol. 2018;69:1088-1098

This article is protected by copyright. All rights reserved 
21) Buch S, Stickel F, Trepo E, Way M, Herrmann A, Nischalke HD, et al. A genome-wide association study confirms PNPLA3 and identifies TM6SF2 and MBOAT7 as risk loci for alcohol-related cirrhosis. Nat Gen 2015; 47: 1443-8

22) He S, McPhaul C, Li JZ, Garuti R, Kinch L, Grishin NV, Cohen JC, et al. A sequence variation (I148M) in PNPLA3 associated with nonalcoholic fatty liver disease disrupts triglyceride hydrolysis. J Biol Chem 2010;285:6706-15

23) Valenti L, Dongiovanni P, Ginanni Corradini S, Burza MA, Romeo S. PNPLA3 I148M variant and hepatocellular carcinoma: a common genetic variant for a rare disease. Dig Liver Dis. 2013;45:619-24

24) Romeo S, Kozlitina J, Xing C, Pertsemlidis A, Cox D, Pennacchio LA, et al. Genetic variation in PNPLA3 confers susceptibility to nonalcoholic fatty liver disease. Nat Genet 2008;40:1461-1465

25) Valenti L, Al-Serri A, Daly AK, Galmozzi E, Rametta R, Dongiovanni P, et al. Homozygosity for the patatin-like phospholipase-3/adiponutrin I148M polymorphism influences liver fibrosis in patients with nonalcoholic fatty liver disease. Hepatology 2010;51:1209-17

26) Valenti L, Rumi M, Galmozzi E, Aghemo A, Del Menico B, De Nicola S, et al. Patatinlike phospholipase domain-containing 3 I148M polymorphism, steatosis, and liver damage in chronic hepatitis C. Hepatology 2011;53:791-9

27) Trépo E, Gustot $T$, Degré D, Lemmers A, Verset L, Demetter P, et al. Common polymorphism in the PNPLA3/adiponutrin gene confers higher risk of cirrhosis and liver damage in alcoholic liver disease. J Hepatol 2011;55:906-12

28) Grimaudo S, Pipitone RM, Pennisi G, Celsa C, Cammà C, Di Marco V, et al. Association Between PNPLA3 rs738409 C>G Variant and Liver-Related Outcomes in Patients with Non-alcoholic Fatty Liver Disease. Clin Gastroenterol Hepatol. 2019 Aug 13. pii: S15423565(19)30886-9. doi: 10.1016/j.cgh.2019.08.011.

29) Stickel F, Buch S, Lau K, Meyer zu Schwabedissen H, Berg T, Ridinger M, et al. Genetic variation in the PNPLA3 gene is associated with alcoholic liver injury in caucasians. Hepatology 2011;53:86-95

This article is protected by copyright. All rights reserved 
30) Trépo E, Pradat P, Potthoff A, Momozawa Y, Quertinmont E, Gustot T, et al. Impact of patatin-like phospholipase-3 (rs738409 $\mathrm{C}>\mathrm{G}$ ) polymorphism on fibrosis progression and steatosis in chronic hepatitis C. Hepatology 2011;54:60-9

31) Kozlitina J, Smagris E, Stender S, Nordestgaard BG, Zhou HH, Tybjærg-Hansen A, et al. Exome-wide association study identifies a TM6SF2 variant that confers susceptibility to nonalcoholic fatty liver disease. Nature Genetics 2014;46:352-6

32) Mahdessian H, Taxiarchis A, Popov S, Silveira A, Franco-Cereceda A, Hamsten A, et al. TM6SF2 is a regulator of liver fat methabolsim influencing triglyceride secretion and hepatic lipid droplet content. Proc Natl Acad Sci USA 2014;111:8913-8

33) Liu YL, Reeves HL, Buurt AD, Tiniakos D, McPherson S, Leathart JB, et al. TM6SF2 rs58542926 influences hepatic fibrosis progression in patients with non-alcoholic fatty liver disease. Nat Commun 2014;5:4309

34) Sookoian S, Castaño GO, Scian R, Mallardi P, Fernández Gianotti T, Burgueño AL, et al. Genetic variation in transmembrane 6 superfamily member 2 and the risk of nonalcoholic fatty liver disease and histological disease severity. Hepatology 2015;61:515-25

35) Holmen OL, Zhang H, Fan Y, Hovelson DH, Schmidt EM, Zhou W, et al. Systematic evaluation of coding variation identifies a candidate causal variant in TM6SF2 influencing total cholesterol and myocardial infarction risk. Nat Genet 2014;46:345-51

36) Dongiovanni P, Petta S, Maglio C, Fracanzani AL, Pipitone R, Mozzi E, et al. Transmembrane 6 superfamily member 2 gene variant disentangles nonalcoholic steatohepatitis from cardiovascular disease. Hepatology 2015;61:506-14

37) Pirola CJ, Sookoian S. The dual and opposite role of the TM6SF2-rs58542926 variant in protecting against cardiovascular disease and conferring risk for nonalcoholic fatty liver: A meta-analysis. Hepatology 2015;62:1742-56

38) Mancina RM, Dongiovanni P, Petta S, Pingitore P, Meroni M, Rametta R, et al. The MBOAT7-TMC4 Variant rs641738 Increases Risk of Nonalcoholic Fatty Liver Disease in Individuals of European Descent. Gastroenterology 2016;150:1219-1230

This article is protected by copyright. All rights reserved 
39) Romano A, Angeli P, Piovesan S, Noventa F, Anastassopoulos G, Chemello L, et al. Newly diagnosed hepatocellular carcinoma in patients with advanced hepatitis $\mathrm{C}$ treated with DAAs: A prospective population study. J Hepatol. 2018;69:345-352

40) Kanwal F, Kramer J, Asch SM, Chayanupatkul M, Cao Y, El-Serag HB. Risk of Hepatocellular Cancer in HCV Patients Treated With Direct-Acting Antiviral Agents. Gastroenterology. 2017;153:996-1005

\section{FIGURE LEGEND}

Figure 1. Cumulative incidence of de novo and recurrent $\mathrm{HCC}$ in the overall $(\mathrm{n}=509)$ population

Figure 2. Cumulative incidence of de novo HCC in patients stratified by sex (2A), presence of diabetes (2B), pre-treatment albumin values (2C), and GRS values (high vs. low) (2D)

Figure 3. Cumulative incidence of de novo $\mathrm{HCC}$ according to presence of two (3A) or three risk factors (3B) among the independent HCC predictors (male sex, diabetes, albumin and GRS score)

Figure 4. Cumulative incidence of recurrent $\mathrm{HCC}$ according to the presence of diabetes $(4 \mathrm{~A})$

Figure 5. Proportion of patients with de novo HCC according to combination of independent risk factors (male gender, diabetes, albumin $\leq 3.5$ and GRS $>0.597$ )

This article is protected by copyright. All rights reserved 
Table 1. Demographic and clinical characteristics of the 509 patients included in the study

\begin{tabular}{|c|c|c|c|}
\hline & $\begin{array}{c}\text { Overall } \\
\text { population } \\
(\mathbf{n}=\mathbf{5 0 9})\end{array}$ & $\begin{array}{l}\text { No HCC } \\
\text { History } \\
(n=452)\end{array}$ & $\begin{array}{c}\text { Previous HCC } \\
\text { History } \\
(\mathbf{n}=57)\end{array}$ \\
\hline Age, years & $64(28-87)$ & $63(28-87)$ & $72(51-86)$ \\
\hline Males & $297(58 \%)$ & $261(58 \%)$ & $36(63 \%)$ \\
\hline Ethnicity: & & & \\
\hline Italian & $471(93 \%)$ & $418(92 \%)$ & $53(93 \%)$ \\
\hline Egyptian & $38(7 \%)$ & $34(8 \%)$ & $4(7 \%)$ \\
\hline $\mathrm{BMI}, \mathrm{Kg} / \mathrm{m}^{2}$ & $25(16-40)$ & $25(16-40)$ & $25(18-36)$ \\
\hline Diabetes & $99(20 \%)$ & $90(20 \%)$ & $9(16 \%)$ \\
\hline HCV genotype $1 b$ & $254(50 \%)$ & $214(47 \%)$ & $40(70 \%)$ \\
\hline HCV-RNA, IU/mL & $\begin{array}{c}589,557 \\
(138-13,333,872)\end{array}$ & $\begin{array}{c}610,666 \\
(138-13,333,872)\end{array}$ & $\begin{array}{c}407,821 \\
(2,337-5,416,303)\end{array}$ \\
\hline ALT, U/L & $71(8-770)$ & $74(8-770)$ & $59(15-209)$ \\
\hline GGT, U/L & $70(11-890)$ & $71(11-890)$ & $67(12-643)$ \\
\hline Bilirubin, mg/dL & $0.9(0.2-6.3)$ & $0.9(0.2-6.3)$ & $0.9(0.3-2.7)$ \\
\hline INR & $1.1(0.8-2.9)$ & $1.1(0.8-2.9)$ & $1.1(0.9-2.2)$ \\
\hline Albumin, g/dL & $4.1(2.4-5.5)$ & $4.1(2.4-5.5)$ & $4.1(2.7-5.0)$ \\
\hline Creatinine, $\mathrm{mg} / \mathrm{dL}$ & $0.8(0.4-10.1)$ & $0.8(0.4-10.1)$ & $0.8(0.5-1.5)$ \\
\hline Platelets, $\times 10^{3} / \mathrm{mL}$ & $115(26-753)$ & $115(26-753)$ & $115(32-471)$ \\
\hline $\mathrm{AFP}, \mathrm{ng} / \mathrm{mL}$ & $10(1-537)$ & $9(1-537)$ & $10(2-192)$ \\
\hline CPT score A & $442(87 \%)$ & $393(87 \%)$ & $49(86 \%)$ \\
\hline Esophageal varices & $182(36 \%)$ & $133(29 \%)$ & $25(44 \%)$ \\
\hline $\mathrm{LSM}, \mathrm{kPa}$ & $19.4(12.0-75.0)$ & $17.4(12.0-75.0)$ & $21.0(12.0-36.3)$ \\
\hline FIB-4 & $4.9(0.3-46.3)$ & $4.9(0.3-46.3)$ & $6.0(1.1-22.4)$ \\
\hline PNPLA3, CG/GG & $275(54 \%)$ & $250(55 \%)$ & $25(44 \%)$ \\
\hline MBOAT7, CT/TT & $363(71 \%)$ & $325(72 \%)$ & $38(67 \%)$ \\
\hline TM6SF2, CT/TT & $46(9 \%)$ & $43(10 \%)$ & $3(5 \%)$ \\
\hline$G C K R, \mathrm{CT} / \mathrm{TT}$ & $380(75 \%)$ & $341(75 \%)$ & $39(68 \%)$ \\
\hline GRS score (IQR) & $0.329(0.128-0.457)$ & $0.329(0.130-0.459)$ & $0.193(0.111-0.394)$ \\
\hline
\end{tabular}


For single genetic variants, we considered carriage of the risk allele.

BMI: body mass index; ALT: alanine aminotransferase; GGT: gamma-glutamyl transpeptidase; INR: international standardized ratio; AFP: alpha-fetoprotein; CPT: ChildPugh-Turcotte score; LSM: liver stiffness measurement; GRS: genetic risk score; IQR: Interquartile Range.

Table 2. Baseline predictors of de novo $\mathrm{HCC}$ at univariate and multivariate Cox regression analysis in 452 cirrhotic patients treated with DAA

\begin{tabular}{|l|c|c|c|c|c|c|}
\hline Variables & Patients & Category & \multicolumn{2}{|c|}{ Univariate Analysis } & \multicolumn{2}{|c|}{ Multivariate Analysis } \\
& number & & HR (95\% CI) & p value & HR (95\% CI) & p value \\
\hline Male gender & 452 & yes $v s$ no & $2.62(1.19-5.76)$ & 0.01 & $2.54(1.15-5.63)$ & 0.02 \\
\hline Diabetes & 452 & yes $v s$ no & $2.83(1.43-5.56)$ & 0.003 & $2.39(1.20-4.74)$ & 0.01 \\
\hline INR, ratio & 451 & continuous & $2.64(1.27-5.48)$ & 0.009 & - & - \\
\hline Albumin, g/dL & 451 & continuous & $0.37(0.20-0.68)$ & 0.001 & $0.35(0.19-0.64)$ & 0.001 \\
\hline
\end{tabular}




\begin{tabular}{|l|c|c|c|c|c|c|}
\hline LSM, kPa & 379 & continuous & $1.04(1.02-1.06)$ & $<0.001$ & - & - \\
\hline FIB-4 & 446 & continuous & $1.06(1.01-1.10)$ & 0.004 & - & - \\
\hline GRS $>0.597$ & 452 & yes $v s$ no & $2.44(1.15-5.20)$ & 0.02 & $2.30(1.03-5.11)$ & 0.04 \\
\hline
\end{tabular}

All variables were analyzed at baseline (DAA start). Variables not statistically significant at univariate analysis: Age (continuous), Ethnicity (Italian vs Egyptian), BMI (continuous), HCV genotype (HCV-1 vs others), HCV-RNA (continuous), ALT (continuous), GGT (continuous), Bilirubin (continuous), platelet count (continuous), creatinine (continuous), esophageal varices (yes vs. no), AFP (continuous), PNPLA3 (CG vs GG), MBOAT7 (CT vs TT), TM6SF2 (CT vs TT), GCKR (CT vs TT) 
Table 3. Baseline predictors of recurrent $\mathrm{HCC}$ at univariate and multivariate Cox regression analysis in 57 cirrhotic patients treated with DAA

\begin{tabular}{|l|c|c|c|c|c|c|}
\hline Variable & Patients & Category & \multicolumn{2}{|c|}{ Univariate Analysis } & \multicolumn{2}{c|}{ Multivariate Analysis } \\
\cline { 3 - 7 } & number & & HR (95\% CI) & p value & HR (95\% CI) & p value \\
\hline Ethnicity & 57 & Italian vs. Egyptian & $0.12(0.34-0.48)$ & 0.002 & $0.22(0.05-0.91)$ & 0.03 \\
\hline BMI, Kg/m & 57 & continuous & $1.10(1.00-1.22)$ & 0.04 & - & - \\
\hline Diabetes & 57 & yes vs.no & $3.57(1.46-8.75)$ & 0.005 & $2.78(1.03-7.44)$ & 0.04 \\
\hline
\end{tabular}

All variables were analyzed at baseline (DAA start). Variables not statistically significant at univariate analysis: Age (continuous), male gender (yes vs. no), HCV genotype (HCV-1 vs others), HCV-RNA (continuous), ALT (continuous), GGT (continuous), Bilirubin (continuous), INR (continuous), albumin (continuous), platelet count (continuous), creatinine (continuous), esophageal varices (yes vs. no), AFP (continuous), LSM (continuous), FIB-4 (continuous), PNPLA3 (CG vs GG), MBOAT7 (CT vs TT), TM6SF2 (CT vs TT), GCKR (CT vs TT), GRS score (continuous), GRS score $<0.191$ (yes vs. no) 


\section{Figure 1}

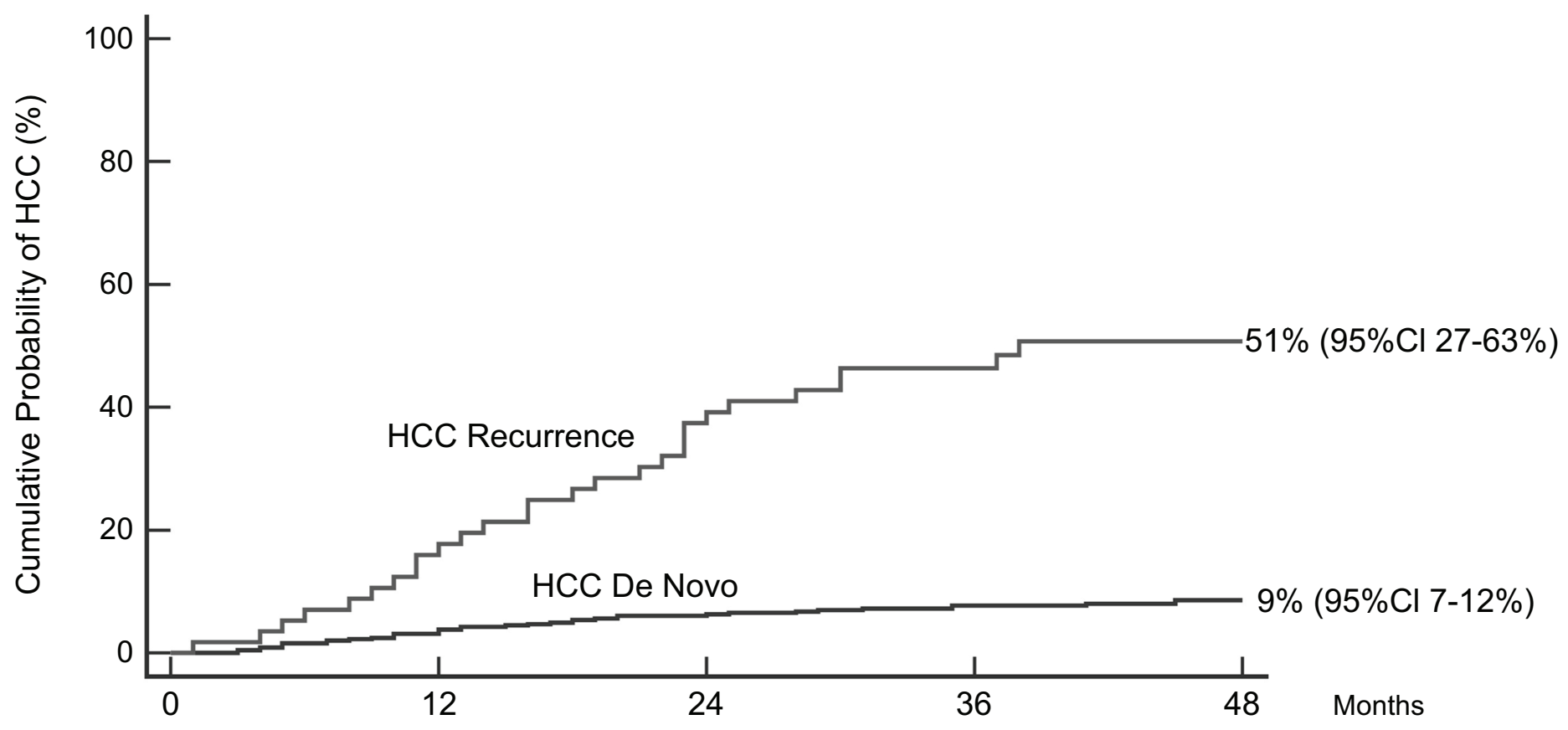

Patients still at risk 452

426

414

367

52

57

46

34

25

3

hep_31500_f1.eps 
Figure 2A

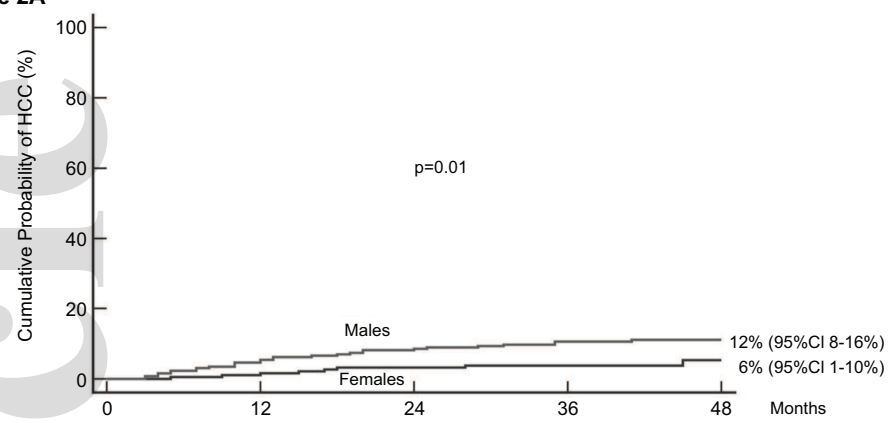

Patients still at risk 191 261

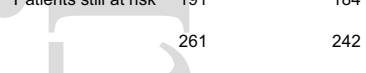

$2 B$

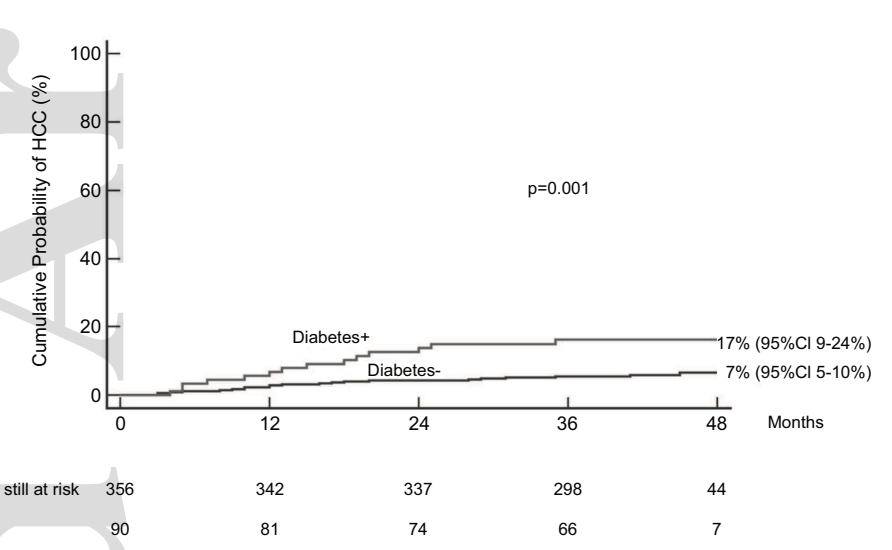

2C

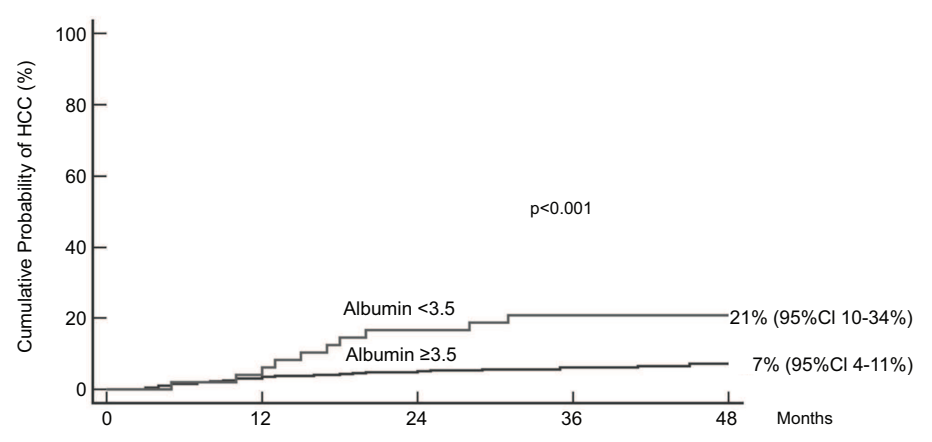

Patients still at risk 400

51

380

45

373

40

333

34

2D

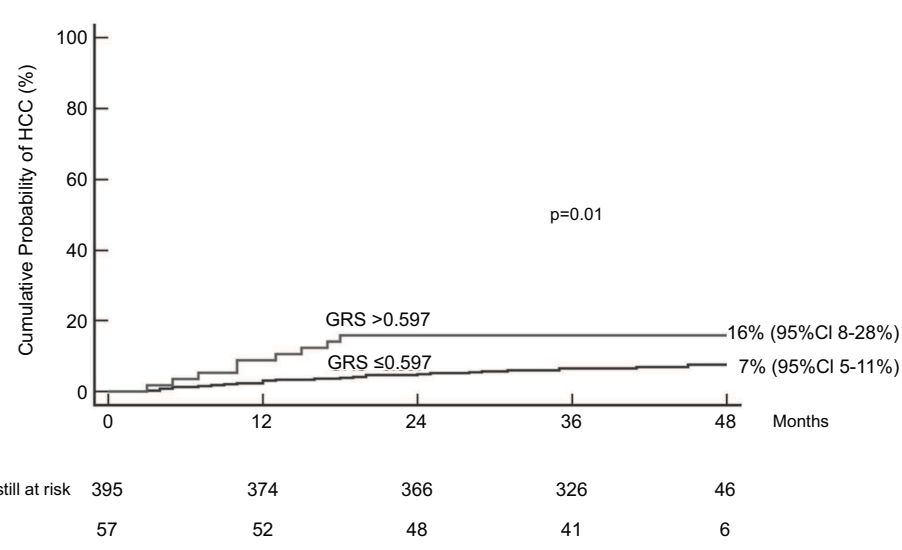

hep_31500_f2.eps 
Figure 3A

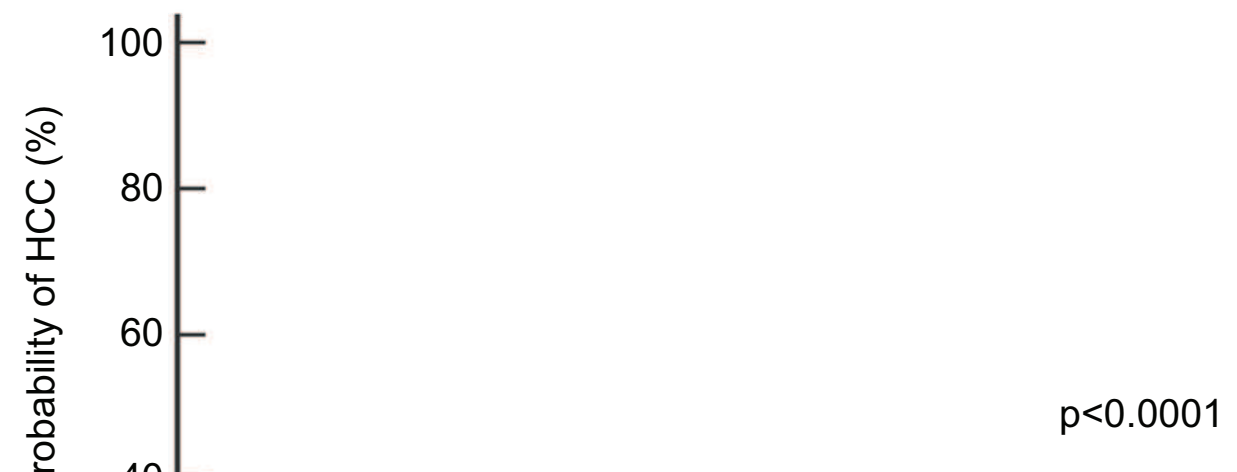

Patients still at risk 332

321

318

285

40

120

105

96

82

12

\section{B}

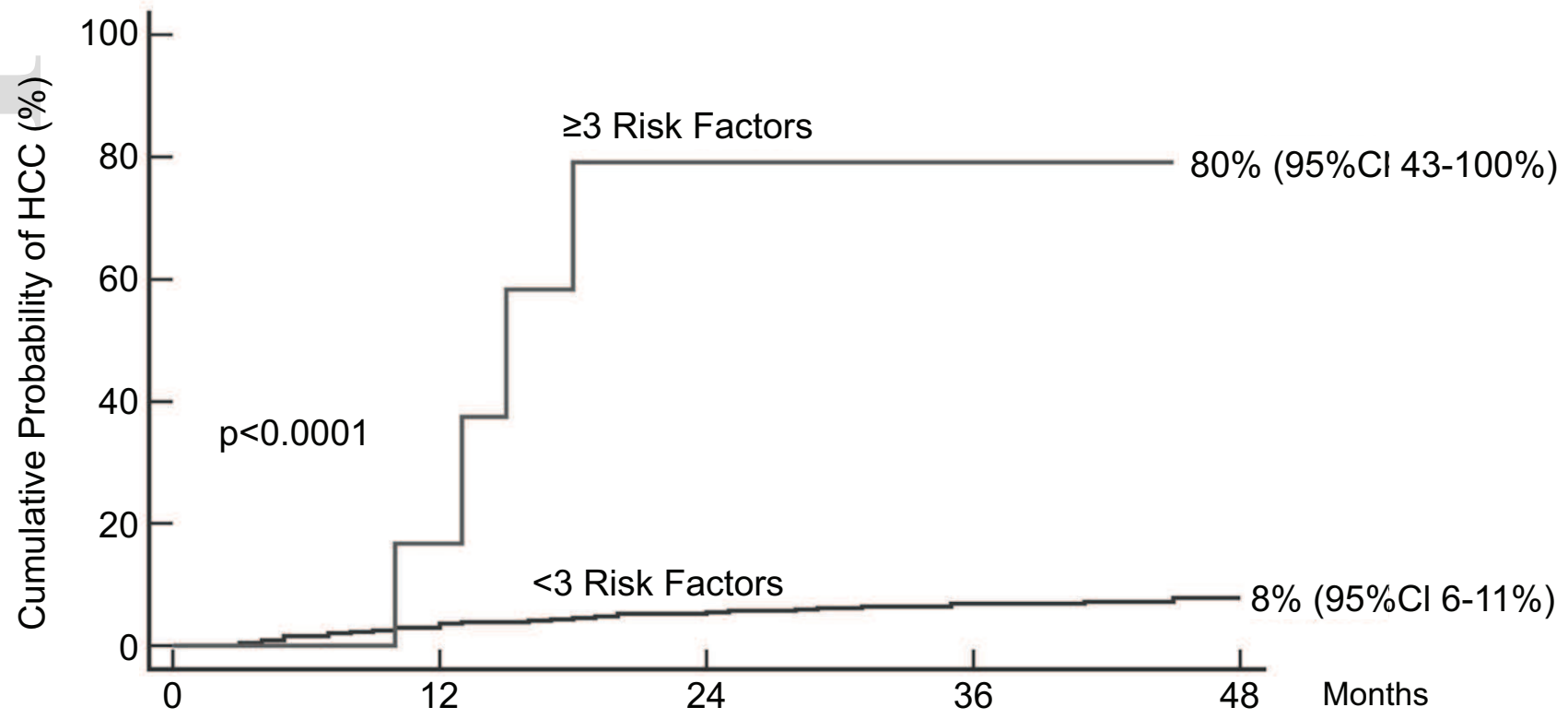


Figure 4

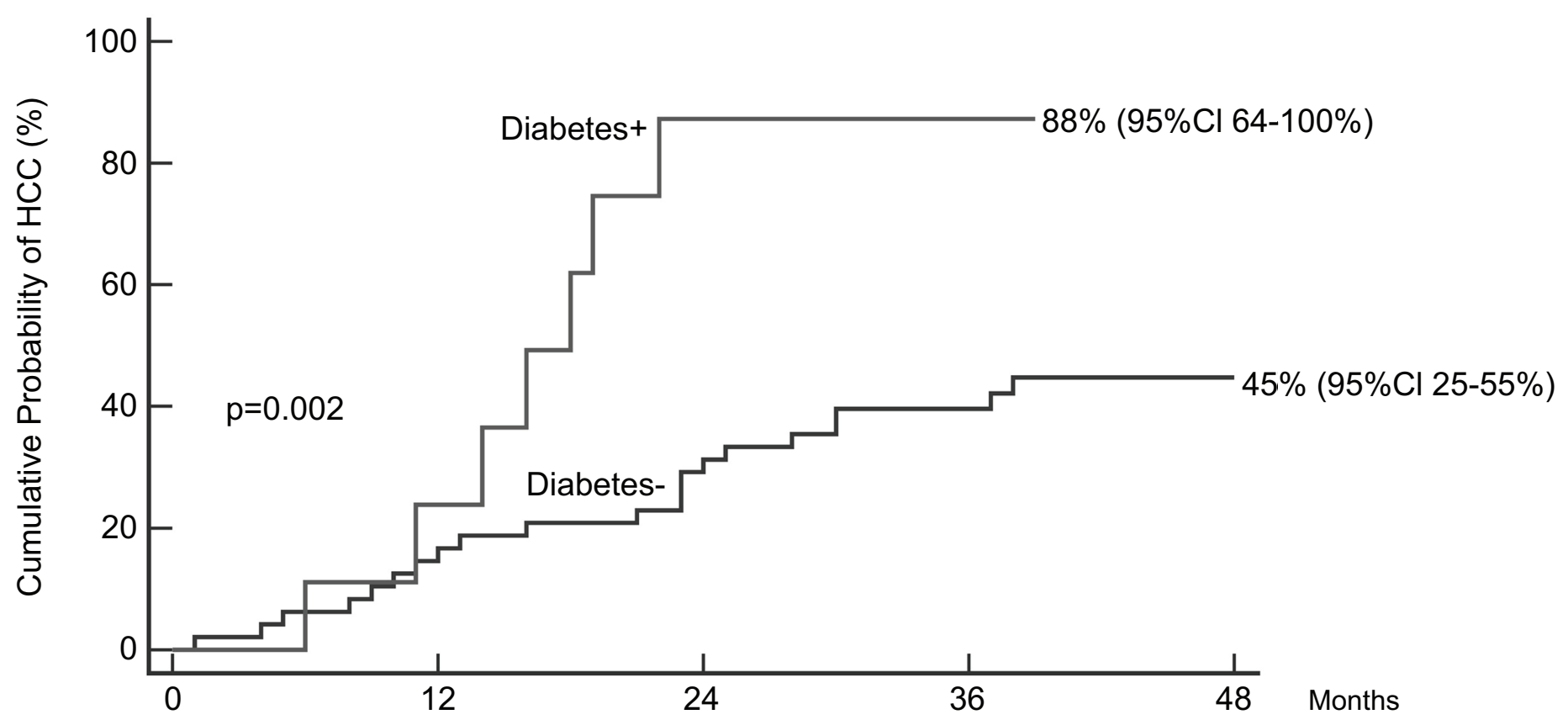

Patients still at risk 48

40

33

24

3

9

6

1

1

0

hep_31500_f4.eps 


\section{Figure 5}

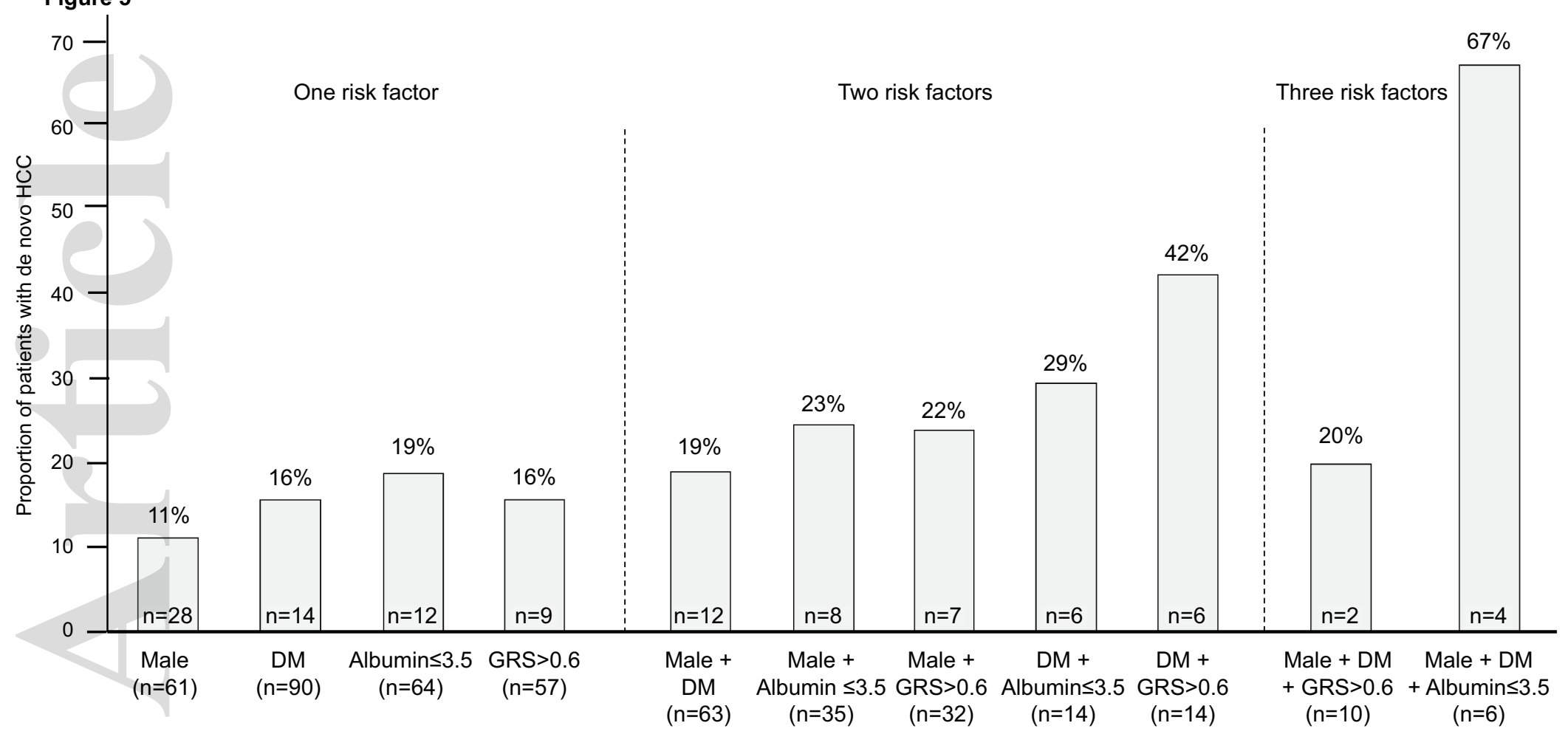

hep_31500_f5.eps 